\title{
Effect of Family Variables on Multiple Intelligences of Secondary School Students of Gujarat State
}

\author{
Dr. Sanjay Kumar M. Gupta ${ }^{1}$
}

\section{ABSTRACT}

There are billions of people in the world, but it is impossible to find two people identical because God doesn't repeat His creation. It means everybody is inborn different. But, our education system is such that treats everybody in more or less same way which hampers the development of a child negatively and his or her contribution as well. Hence, researcher has conducted this study entitled "Effect of Family Variables on Multiple Intelligences of Secondary School Students of Gujarat State" to study the individual potential of children in terms of their intelligences and the effect of family related variables on their intelligences. It was found that some of the family and environment related variables affect the intelligences of learner positively and some do not have any effect as given.

Keywords: Family, Variables, Intelligence, Secondary School, Students, Gujarat State.

Gardner (1999) emphasized on 'diversity of students' intelligence and acknowledged that every child possesses inborn creativity, but many children lose interests in learning due to rigid educational curriculum that is only focused on verbal/linguistic and Logical Mathematical intelligence. In order to develop each child's uniqueness, educators should be able to look at the inner world of children. Teachers need to be aware of these differences. Insensitivity of teachers and trainers toward these unique ways of thinking and learning may end up students being labeled as underachievers.

To understand these diversities of students, Gardner (1993) identified seven different intelligences or seven different ways that a person can learn. They were 1 to 7 in the list given below. Later, Dr. Gardner (1999) proposed two more different intelligences to account for a broader range of human potential in children and adults. Following intelligences were suggested by Gardner.

\footnotetext{
${ }^{1}$ Lecturer in Selection Grade, Dept of Education, Kadi Sarva Vishwavidyalaya, Gandhinagar- Gujarat *Responding Author (C) 2016 I S Gupta; licensee IJIP. This is an Open Access Research distributed under the terms of the Creative Commons Attribution License (http://creativecommons.org/licenses/by/2.0), which permits unrestricted use, distribution, and reproduction in any Medium, provided the original work is properly cited.
} 
1. Linguistic intelligence

3. Spatial intelligence

5. Musical intelligence

7. Intrapersonal intelligence
2. Logical-Mathematical intelligence

4. Bodily-Kinesthetic intelligence

6. Interpersonal intelligence

8. Naturalist intelligence

Hence, it is essential to understand learners from their point of view. Moreover, apart from inherited potential, learners are also affected environment they face including working status and qualification of the parents, number of siblings and nature of family they live in. Hence, in order to understand the learners' individuality and the effect of family related variables on their intelligences, this research has been carried out. Findings of this research provide the base to teachers, parents, schools, curriculum developers, and policy makers etc. to take decisions for next generation.

\section{REVIEW OF PREVIOUS RESEARCHES}

The researcher has reviewed the previous researches to conduct the present research. Out of total 23 studies, 9 studies were found conducted in the foreign settings and 14 were from Indian settings. The Multiple Intelligences were used as dependent variable in all of the studies. Moreover, some of the other variables were studied in reference to the Multiple Intelligences. Among them Maria do Rozario (2003) analysed the MI theory in English Language Teaching (ELT); Gogebakan, Derya (2003) studied how MI differ in terms of Grade level and Gender and Gurçay, Deniz (2003) analysed the effect of Multiple Intelligences based instruction on students' Physics achievement; Seyyed Ayatollah Razmjoo (2008) studied language mastery and gender; Aysel Sarisaoglu and Arda Arikan(2009) studied gender and parental education; HassanPasha Sharifi (2005) studied gender and achievement level; Rio Sumarini and others studied achievement level. Ramzi Nasser, \& other (2008), and Sudha Chikara (2008) studied gender; Sawlis, Caryn (2009) studied the importance of adding MI to virtual learning; Gale, La Tonya (2012) studied How Gardner's MI theory influenced the leadership in organisation; Jamal and Fadi (2012) studied the level of Multiple Intelligences in Social science teachers in Jordan; Jose Agnes (2011) analysed the relation between academic achievement and Bodily-Kinesthetic of school children; Partani, Swati (2011) studied to develop MI training module for Preschool teacher and analysed its effect on Preschool children; Ashok G. Chanchu (2012) studied Residential area and subject stream as the variables.

\section{OBJECTIVES OF THE STUDY}

The study was carried out to realize following objectives.

1. To study the effect of working status of parents on various Multiple Intelligences of students

2. To study the effect of educational qualification of parents on various Multiple Intelligences of students

3. To compare the Multiple Intelligences of students residing in joint family and nuclear family

4. To study the effect of number of siblings on various Multiple Intelligences of students. 


\section{Null Hypotheses:}

1. There is no significant difference in mean scores of Interpersonal intelligence of P1 and P2 children.

2. There is no significant difference in mean scores of Intrapersonal intelligence of P1 and P2 children.

3. There is no significant difference in mean scores of Linguistic intelligence of P1 and P2 children.

4. There is no significant difference in mean scores of Logical Mathematical intelligence of P1 and P2 children.

5. There is no significant difference in mean scores of Naturalist intelligence of P1 and P2 children.

6. There is no significant difference in mean scores of Spatial intelligence of P1 and P2 children.

7. There is no significant difference in mean scores of Bodily Kinesthetic intelligence of P1 and P2 children.

8. There is no significant difference in mean scores of Musical intelligence of P1 and P2 children.

9. There is no significant difference in mean scores of Interpersonal intelligence of Q1 and Q2 children.

10. There is no significant difference in mean scores of Intrapersonal intelligence of Q1 and Q2 children

11. There is no significant difference in mean scores of Linguistic intelligence of Q1 and Q2 children.

12. There is no significant difference in mean scores of Logical Mathematical intelligence of Q1 and Q2 children.

13. There is no significant difference in mean scores of Naturalist intelligence of Q1 and Q2 children.

14. There is no significant difference in mean scores of Spatial intelligence of Q1 and Q2 children.

15. There is no significant difference in mean scores of Bodily Kinesthetic intelligence of Q1 and Q2 children.

16. There is no significant difference in mean scores of Musical intelligence of Q1 and Q2 children.

17. There is no significant difference in mean scores of Interpersonal intelligence of students staying in Joint family and Nuclear family.

18. There is no significant difference in mean scores of Intrapersonal intelligence of students staying in Joint family and Nuclear family.

19. There is no significant difference in mean scores of Linguistic intelligence of students staying in Joint family and Nuclear family. 
20. There is no significant difference in mean scores of Logical Mathematical intelligence of students staying in Joint family and Nuclear family.

21. There is no significant difference in mean scores of Naturalist intelligence of students staying in Joint family and Nuclear family.

22. There is no significant difference in mean scores of Spatial intelligence of students staying in Joint family and Nuclear family.

23. There is no significant difference in mean scores of Bodily Kinesthetic intelligence of students staying in Joint family and Nuclear family.

24. There is no significant difference in mean scores of Musical intelligence of students staying in Joint family and Nuclear family.

25. There is no significant difference in mean scores of Interpersonal intelligence of students 'without siblings' and 'with siblings'.

26. There is no significant difference in mean scores of Intrapersonal intelligence of students 'without siblings' and 'with siblings'.

27. There is no significant difference in mean scores of Linguistic intelligence of students 'without siblings' and 'with siblings'.

28. There is no significant difference in mean scores of Logical Mathematical intelligence of students 'without siblings' and 'with siblings'.

29. There is no significant difference in mean scores of Naturalist intelligence of students 'without siblings' and 'with siblings'.

30. There is no significant difference in mean scores of Spatial intelligence of students 'without siblings' and 'with siblings'.

31. There is no significant difference in mean scores of Bodily Kinesthetic intelligence of students 'without siblings' and 'with siblings'.

32. There is no significant difference in mean scores of Musical intelligence of students 'without siblings' and 'with siblings'.

\section{DELIMITATIONS}

\section{Following were the delimitations of the present study:}

The study was delimited to the students of Std. IX \& X of English Medium Schools of Gujarat State.

\section{Operational Definitions of Key Terms}

Multiple Intelligences: Total eight intelligences as given here are called as Multiple Intelligences. They are: Linguistic Intelligence, Logical-Mathematical intelligence, Spatial intelligence, Bodily-kinaesthetic intelligence, Musical intelligence, Interpersonal intelligence, Intrapersonal intelligence and Naturalist intelligence. Scores obtained on the statements of each intelligence are the scores of the student on that intelligence. 
Working status of Parents: It refers to whether one of both the parents is working or both of them are working for economic purpose.

P1 Family: It refers to one of the both parents is carrying out economic activity for family sustenance.

P2 Family: It refers to both of the parents are carrying out economic activity for family sustenance.

Siblings: It refers to the real (blood relation) sister and brother of a student.

Nature of Family: It refers to whether the student is staying in Joint family or Nuclear family.

Graduate Parents: One or both of the parents are Graduate.

Non Graduate Parents: None of the parents is Graduate.

\section{Variables}

The variables undertaken in the present study were as given below:

a. Dependent Variables:

1. Multiple Intelligences

i. Interpersonal intelligence

ii. Intrapersonal intelligence

iii. Linguistic Intelligence

iv. Logical-Mathematical intelligence

v. Naturalist intelligence

vi. Spatial intelligence

vii. Bodily-kinaesthetic intelligence

viii. Musical intelligence

\section{b. Independent Variables:}

1. Working status of Parents -

2. Nature of Family -

One working and both working parents

Joint and Nuclear

3. Siblings -

4. Qualification of Parents -

'With Siblings' and Without Siblings

Both/One Graduate \& None-graduate

c. Control Variables:

1. Gujarat State

2. English Medium Schools

3. Standard IX \& X Students and Teachers

4. GSEB (Gujarat Secondary Education Board), CBSE (Central Board of Secondary Education) and CISCE (Council of Indian School Certificate Examinations) schools

\section{Population}

All STD IX and X class students studying in English Medium Schools of Gujarat State constitute the population of the study.

\section{Sample}

Out of 30 districts of Gujarat state, 15 districts were randomly selected for collecting data from 4417 students. In case of up to 2 sections of the same class, one section of each standard i.e. IX and $\mathrm{X}$ was taken in the sample. In case of more than two sections i.e. 3 or more sections in the 
same standard, two sections were selected randomly through lottery and all the students of those sections were included in the sample and administered Multiple Intelligences Scale to respond.

\section{RESEARCH METHOD}

It is a survey research, because it assesses the present status of Multiple Intelligences of secondary school students of Gujarat State.

\section{Research Tool}

\section{Tool: Multiple Intelligences Scale}

A five point Multiple Intelligences Scale constructed through adopting Likert type scale procedure. Weightage given for scoring was: 5 for Always, 4 for usually, 3 for Sometimes, 2 for Hardly, and 1 for Never. Following steps were followed in its construction.

1) Construction of Items and Finalization of the First Draft

2) Editing, Pre Piloting for Finalization of Second Draft

3) Experts' feedback

4) Third draft of Multiple Intelligences Scale

5) Piloting and Finalization of Multiple Intelligences Scale

\section{Data Collection}

After the prior approval of the school authorities, data was collected from different schools. Multiple Intelligences Scale was administered on 4417 students. It required patience and persistence in data collection, as many of the schools did not co operate in the beginning, but finally they consented due to constant efforts and counselling. It required a lot of efforts to convince the schools that the data collected from the schools would be used exclusively for research purpose and no institution or individual results would be drawn, declared and published.

\section{Data Analysis}

Being a quantitative research, data was inserted in Micro Soft Excel Sheet as per the classification based on hypotheses. It was analyzed with the help of $t$ test. 


\section{MAJOR FINDINGS AND DISCUSSION:}

\section{FOR OBJECTIVE-1}

$\mathbf{P 1}=$ Either father or mother is working is working to earn money P2= Both father and mother are working to earn money

Table No: 1. Parents' Working Status Wise Comparison of Mean, S.D. and C.R. Values of Multiple Intelligences

\begin{tabular}{|c|c|c|c|c|c|c|c|}
\hline Intelligences & $\begin{array}{l}\text { Working Status } \\
\text { of Parents }\end{array}$ & Mean & $\mathbf{N}$ & S.D. & $\mathrm{SE}_{\mathbf{D}}$ & C.R. & Remark \\
\hline \multirow{2}{*}{$\begin{array}{l}\text { HO1 } \\
\text { Interpersonal }\end{array}$} & P1 Children & 48.88 & 3731 & 6.72 & \multirow[t]{2}{*}{0.279} & \multirow[t]{2}{*}{0.401} & Not \\
\hline & P2 Children & 48.99 & 686 & 6.73 & & & $\begin{array}{l}\text { Significant at } \\
0.05 \text { level }\end{array}$ \\
\hline \multirow{2}{*}{$\begin{array}{l}\text { HO2 } \\
\text { Intrapersonal }\end{array}$} & P1 Children & 43.84 & 3731 & 6.86 & \multirow[t]{2}{*}{0.285} & \multirow[t]{2}{*}{1.86} & Not \\
\hline & P2 Children & 44.37 & 686 & 6.86 & & & $\begin{array}{l}\text { Significant at } \\
0.05 \text { level }\end{array}$ \\
\hline \multirow{2}{*}{$\begin{array}{l}\text { HO3 } \\
\text { Linguistic }\end{array}$} & P1 Children & 44.65 & 3731 & 7.37 & \multirow[t]{2}{*}{0.311} & \multirow[t]{2}{*}{2.38} & \multirow{2}{*}{$\begin{array}{l}\text { Significant at } \\
0.05 \text { level }\end{array}$} \\
\hline & P2 Children & 45.39 & 686 & 7.52 & & & \\
\hline \multirow{2}{*}{$\begin{array}{l}\text { HO } 4 \text { Logical } \\
\text { Mathematical }\end{array}$} & P1 Children & 47.03 & 3731 & 7.63 & \multirow[t]{2}{*}{0.322} & \multirow[t]{2}{*}{0.333} & Not \\
\hline & P2 Children & 47.14 & 686 & 7.79 & & & Significant \\
\hline \multirow{2}{*}{$\begin{array}{l}\text { HO } 5 \\
\text { Naturalist }\end{array}$} & P1 Children & 47.61 & 3731 & 7.87 & \multirow[t]{2}{*}{0.333} & \multirow[t]{2}{*}{0.133} & Not \\
\hline & P2 Children & 47.56 & 686 & 8.05 & & & $\begin{array}{l}\text { Significant at } \\
0.05 \text { level }\end{array}$ \\
\hline \multirow{2}{*}{$\begin{array}{l}\text { HO } 6 \\
\text { Spatial }\end{array}$} & P1 Children & 48.19 & 3731 & 6.88 & \multirow[t]{2}{*}{0.291} & \multirow[t]{2}{*}{0.907} & Not \\
\hline & P2 Children & 48.46 & 686 & 7.03 & & & $\begin{array}{l}\text { Significant at } \\
0.05 \text { level }\end{array}$ \\
\hline \multirow{2}{*}{$\begin{array}{l}\text { HO } 7 \text { Bodily } \\
\text { Kinesthetic }\end{array}$} & P1 Children & 48.34 & 3731 & 6.86 & \multirow[t]{2}{*}{0.286} & \multirow[t]{2}{*}{2.08} & \multirow{2}{*}{$\begin{array}{l}\text { Significant at } \\
0.05 \text { level }\end{array}$} \\
\hline & P2 Children & 48.94 & 686 & 6.90 & & & \\
\hline \multirow{2}{*}{$\begin{array}{l}\text { HO } 8 \\
\text { Musical }\end{array}$} & P1 Children & 47.37 & 3731 & 8.70 & \multirow[t]{2}{*}{0.364} & \multirow[t]{2}{*}{0.627} & \multirow{2}{*}{$\begin{array}{l}\text { Not } \\
\text { Significant at } \\
0.05 \text { level }\end{array}$} \\
\hline & P2 Children & 47.60 & 686 & 8.77 & & & \\
\hline
\end{tabular}

It was found that children belonging to the family in which both mother and father are working as earning member, were found better in Linguistic Intelligence as well as in Bodily Kinesthetic Intelligence than the children belonging to the family in which either mother or father is only working. It was also drawn from the data that the rest of the intelligences are not affected by the working status of parents. 
FOR OBJECTIVE-2

Q1= both or one of the two parents is graduate or higher qualified

Q2= none of the two parents is graduate

Table No. 2 Parents' Qualification Wise Comparison of Mean, S.D. and C.R. Values of Multiple Intelligences

\begin{tabular}{|c|c|c|c|c|c|c|c|}
\hline Intelligence & $\begin{array}{l}\text { Parents' } \\
\text { Qualification }\end{array}$ & Mean & $\mathbf{N}$ & S.D. & $\mathrm{SE}_{\mathbf{D}}$ & C.R. & Remark \\
\hline \multirow{2}{*}{$\begin{array}{l}\text { HO } 9 \\
\text { Interpersonal }\end{array}$} & Q2(None Graduate) & 48.83 & 1503 & 6.87 & \multirow[t]{2}{*}{0.216} & \multirow[t]{2}{*}{0.465} & \multirow{2}{*}{$\begin{array}{ll}\text { Not } & \\
\text { Significant } \\
\text { at } \quad 0.05 \\
\text { level }\end{array}$} \\
\hline & $\begin{array}{l}\text { Q1 (One or both are } \\
\text { Graduate or higher } \\
\text { qualified) }\end{array}$ & 48.93 & 2914 & 6.64 & & & \\
\hline \multirow{2}{*}{$\begin{array}{l}\text { HO } 10 \\
\text { Intrapersonal }\end{array}$} & Q2(None Graduate) & 43.55 & 1503 & 6.74 & \multirow[t]{2}{*}{0.215} & \multirow[t]{2}{*}{2.57} & \multirow{2}{*}{$\begin{array}{l}\text { Significant } \\
\text { at } \quad 0.05 \\
\text { level }\end{array}$} \\
\hline & $\begin{array}{l}\text { Q1 (One or both are } \\
\text { Graduate or higher } \\
\text { qualified) }\end{array}$ & 44.10 & 2914 & 6.84 & & & \\
\hline \multirow{2}{*}{$\begin{array}{l}\text { HO } 11 \\
\text { Linguistic }\end{array}$} & Q2(None Graduate) & 44.44 & 1503 & 7.48 & \multirow[t]{2}{*}{0.235} & \multirow[t]{2}{*}{2.01} & \multirow{2}{*}{$\begin{array}{l}\text { Significant } \\
\text { at } \quad 0.05 \\
\text { level }\end{array}$} \\
\hline & $\begin{array}{l}\text { Q1 (One or both are } \\
\text { Graduate or higher } \\
\text { qualified) }\end{array}$ & 44.92 & 2914 & 7.25 & & & \\
\hline \multirow{2}{*}{$\begin{array}{l}\text { HO } 12 \\
\text { Logical } \\
\text { Mathematical }\end{array}$} & Q2(None Graduate) & 47.77 & 1503 & 7.71 & \multirow[t]{2}{*}{0.241} & \multirow[t]{2}{*}{1.50} & \multirow{2}{*}{$\begin{array}{l}\text { Not } \\
\text { Significant } \\
\text { at } \quad 0.05 \\
\text { level }\end{array}$} \\
\hline & $\begin{array}{l}\text { Q1 (One or both are } \\
\text { Graduate or higher } \\
\text { qualified) }\end{array}$ & 49.06 & 2914 & 7.36 & & & \\
\hline \multirow{2}{*}{$\begin{array}{l}\text { HO } 13 \\
\text { Naturalist }\end{array}$} & Q2(None Graduate) & 47.72 & 1503 & 7.69 & \multirow[t]{2}{*}{0.243} & \multirow[t]{2}{*}{1.14} & \multirow{2}{*}{$\begin{array}{l}\text { Not } \\
\text { Significant } \\
\text { at } \quad 0.05 \\
\text { level }\end{array}$} \\
\hline & $\begin{array}{l}\text { Q1 (One or both are } \\
\text { Graduate or higher } \\
\text { qualified) }\end{array}$ & 47.44 & 2914 & 7.57 & & & \\
\hline \multirow{2}{*}{$\begin{array}{l}\text { HO } 14 \\
\text { Spatial }\end{array}$} & Q2(None Graduate) & 48.06 & 1503 & 6.80 & \multirow[t]{2}{*}{0.218} & \multirow[t]{2}{*}{1.21} & \multirow{2}{*}{$\begin{array}{l}\text { Not } \\
\text { Significant } \\
\text { at } \quad 0.05 \\
\text { level }\end{array}$} \\
\hline & $\begin{array}{l}\text { Q1 (One or both are } \\
\text { Graduate or higher } \\
\text { qualified) }\end{array}$ & 48.32 & 2914 & 6.95 & & & \\
\hline \multirow{2}{*}{$\begin{array}{l}\text { HO } 15 \\
\text { Bodily } \\
\text { Kinesthetic }\end{array}$} & Q2(None Graduate) & 48.52 & 1503 & 6.85 & \multirow[t]{2}{*}{0.218} & \multirow[t]{2}{*}{0.557} & \multirow{2}{*}{$\begin{array}{l}\text { Not } \\
\text { Significant } \\
\text { at } \quad 0.05 \\
\text { level }\end{array}$} \\
\hline & $\begin{array}{l}\text { Q1 (One or both are } \\
\text { Graduate or higher } \\
\text { qualified) }\end{array}$ & 48.40 & 2914 & 6.88 & & & \\
\hline \multirow{2}{*}{$\begin{array}{l}\text { HO } 16 \\
\text { Musical }\end{array}$} & Q2(None Graduate) & 46.98 & 1503 & 8.88 & \multirow[t]{2}{*}{2.279} & \multirow[t]{2}{*}{2.232} & \multirow{2}{*}{$\begin{array}{l}\text { Significant } \\
\text { at } \quad 0.05 \\
\text { level }\end{array}$} \\
\hline & $\begin{array}{l}\text { Q1 (One or both are } \\
\text { Graduate or higher } \\
\text { qualified) }\end{array}$ & 47.20 & 2914 & 8.61 & & & \\
\hline
\end{tabular}


It was found that the students belonging to the family of Graduate parents were better than the students belonging to the Non Graduate Parents in Intrapersonal, Linguistic and Musical Intelligence.

It was also found that the students belonging to the family of Non Graduate parents were not found better than students belonging to the 'Graduate Parents' Family in any of the Intelligences. Moreover, it was also drawn that the qualification of parents did not have any effect of Interpersonal, Logical Mathematical, Naturalist, Spatial and Bodily Kinesthetic Intelligence.

FOR OBJECTIVE-3

Table No. 3: Nature of Family Wise Comparison of Mean, S.D. and C.R. Values of Multiple Intelligences

\begin{tabular}{|c|c|c|c|c|c|c|c|}
\hline Intelligences & $\begin{array}{l}\text { Nature of } \\
\text { Family }\end{array}$ & Mean & $\mathbf{N}$ & S.D. & $\mathrm{SE}_{\mathrm{D}}$ & C.R. & Remark \\
\hline \multirow{2}{*}{$\begin{array}{l}\text { HO } 17 \\
\text { Interpersonal }\end{array}$} & Joint Family & 48.85 & 1911 & 6.79 & \multirow[t]{2}{*}{0.205} & \multirow[t]{2}{*}{0.355} & Not \\
\hline & $\begin{array}{l}\text { Nuclear } \\
\text { Family }\end{array}$ & 49.93 & 2506 & 6.67 & & & $\begin{array}{l}\text { Significant at } \\
0.05 \text { level }\end{array}$ \\
\hline \multirow{2}{*}{$\begin{array}{l}\text { HO } 18 \\
\text { Intrapersonal }\end{array}$} & Joint Family & 44.14 & 1911 & 6.82 & \multirow[t]{2}{*}{0.209} & \multirow[t]{2}{*}{1.870} & Not \\
\hline & $\begin{array}{l}\text { Nuclear } \\
\text { Family }\end{array}$ & 43.75 & 2506 & 7.55 & & & $\begin{array}{l}\text { Significant at } \\
0.05 \text { level }\end{array}$ \\
\hline \multirow{2}{*}{$\begin{array}{l}\text { HO } 19 \\
\text { Linguistic }\end{array}$} & Joint Family & 44.75 & 1911 & 7.55 & \multirow[t]{2}{*}{0.30} & \multirow[t]{2}{*}{0.111} & Not \\
\hline & $\begin{array}{l}\text { Nuclear } \\
\text { Family }\end{array}$ & 47.12 & 2506 & 7.28 & & & $\begin{array}{l}\text { Significant at } \\
0.05 \text { level }\end{array}$ \\
\hline \multirow{2}{*}{$\begin{array}{l}\text { HO } 20 \text { Logical } \\
\text { Mathematical }\end{array}$} & Joint Family & 47.12 & 1911 & 7.96 & \multirow[t]{2}{*}{0.235} & \multirow[t]{2}{*}{0.575} & Not \\
\hline & $\begin{array}{l}\text { Nuclear } \\
\text { Family }\end{array}$ & 46.99 & 2506 & 7.42 & & & $\begin{array}{l}\text { Significant at } \\
0.05 \text { level }\end{array}$ \\
\hline \multirow{2}{*}{$\begin{array}{l}\text { HO } 21 \\
\text { Naturalist }\end{array}$} & Joint Family & 47.70 & 1911 & 7.99 & \multirow[t]{2}{*}{0.240} & \multirow[t]{2}{*}{0.733} & Not \\
\hline & $\begin{array}{l}\text { Nuclear } \\
\text { Family }\end{array}$ & 47.52 & 2506 & 7.83 & & & $\begin{array}{l}\text { Significant at } \\
0.05 \text { level }\end{array}$ \\
\hline \multirow{2}{*}{$\begin{array}{l}\text { HO } 22 \\
\text { Spatial }\end{array}$} & Joint Family & 48.19 & 1911 & 7.02 & \multirow[t]{2}{*}{0.211} & \multirow[t]{2}{*}{0.340} & Not \\
\hline & $\begin{array}{l}\text { Nuclear } \\
\text { Family }\end{array}$ & 48.26 & 2506 & 6.82 & & & $\begin{array}{l}\text { Significant at } \\
0.05 \text { level }\end{array}$ \\
\hline \multirow{2}{*}{$\begin{array}{l}\text { HO } 23 \text { Bodily } \\
\text { Kinesthetic }\end{array}$} & Joint Family & 48.50 & 1911 & 6.92 & \multirow[t]{2}{*}{0.209} & \multirow[t]{2}{*}{0.557} & Not \\
\hline & $\begin{array}{l}\text { Nuclear } \\
\text { Family }\end{array}$ & 48.39 & 2506 & 6.82 & & & $\begin{array}{l}\text { Significant at } \\
0.05 \text { level }\end{array}$ \\
\hline \multirow{2}{*}{$\begin{array}{l}\text { HO } 24 \\
\text { Musical }\end{array}$} & Joint Family & 47.23 & 1911 & 8.62 & \multirow[t]{2}{*}{0.264} & \multirow[t]{2}{*}{1.125} & Not \\
\hline & $\begin{array}{l}\text { Nuclear } \\
\text { Family }\end{array}$ & 47.53 & 2506 & 8.77 & & & $\begin{array}{l}\text { Significant at } \\
0.05 \text { level }\end{array}$ \\
\hline
\end{tabular}


It was found that nature of family did not have any effect on any of the intelligences of students.

FOR OBJECTIVE-4

Table No. 4: Sibling Wise Comparison of Mean, S.D. and C.R. Values of Multiple Intelligences

\begin{tabular}{|c|c|c|c|c|c|c|c|}
\hline Intelligences & $\begin{array}{ll}\text { Status of } \\
\text { Siblings }\end{array}$ & Mean & $\mathbf{N}$ & S.D. & $S E_{D}$ & C.R. & Remark \\
\hline \multirow[t]{2}{*}{$\begin{array}{l}\text { HO } 25 \\
\text { Interpersonal }\end{array}$} & $\begin{array}{l}\text { Without } \\
\text { Siblings }\end{array}$ & 47.73 & 69 & 10.34 & \multirow[t]{2}{*}{1.027} & \multirow[t]{2}{*}{0.97} & \multirow[t]{2}{*}{$\begin{array}{l}\text { Not Significant } \\
\text { at } 0.05 \text { level }\end{array}$} \\
\hline & $\begin{array}{l}\text { With } \\
\text { Siblings }\end{array}$ & 48.90 & 4348 & 6.71 & & & \\
\hline \multirow[t]{2}{*}{$\begin{array}{l}\text { HO } 26 \\
\text { Intrapersonal }\end{array}$} & $\begin{array}{l}\text { Without } \\
\text { Siblings }\end{array}$ & 42.29 & 69 & 6.54 & \multirow[t]{2}{*}{0.794} & \multirow[t]{2}{*}{2.08} & \multirow[t]{2}{*}{$\begin{array}{l}\text { Significant at } \\
0.05 \text { level }\end{array}$} \\
\hline & $\begin{array}{l}\text { With } \\
\text { Siblings }\end{array}$ & 43.94 & 4348 & 6.81 & & & \\
\hline \multirow[t]{2}{*}{$\begin{array}{l}\text { HO } 27 \\
\text { Linguistic }\end{array}$} & $\begin{array}{l}\text { Without } \\
\text { Siblings }\end{array}$ & 43.28 & 69 & 7.49 & \multirow[t]{2}{*}{0.012} & \multirow[t]{2}{*}{0.251} & \multirow[t]{2}{*}{$\begin{array}{l}\text { Not Significant } \\
\text { at } 0.05 \text { level }\end{array}$} \\
\hline & $\begin{array}{l}\text { With } \\
\text { Siblings }\end{array}$ & 46.75 & 4348 & 7.32 & & & \\
\hline \multirow[t]{2}{*}{$\begin{array}{l}\text { HO } 28 \text { Logical } \\
\text { Mathematical }\end{array}$} & $\begin{array}{l}\text { Without } \\
\text { Siblings }\end{array}$ & 46.75 & 69 & 8.21 & \multirow[t]{2}{*}{0.995} & \multirow[t]{2}{*}{0.255} & \multirow[t]{2}{*}{$\begin{array}{l}\text { Not Significant } \\
\text { at } 0.05 \text { level }\end{array}$} \\
\hline & $\begin{array}{l}\text { With } \\
\text { Siblings }\end{array}$ & 47.00 & 4348 & 7.47 & & & \\
\hline \multirow[t]{2}{*}{$\begin{array}{l}\text { HO } 29 \\
\text { Naturalist }\end{array}$} & $\begin{array}{l}\text { Without } \\
\text { Siblings }\end{array}$ & 47.91 & 69 & 7.52 & \multirow[t]{2}{*}{0.913} & \multirow[t]{2}{*}{0.421} & \multirow[t]{2}{*}{$\begin{array}{l}\text { Not Significant } \\
\text { at } 0.05 \text { level }\end{array}$} \\
\hline & $\begin{array}{l}\text { With } \\
\text { Siblings }\end{array}$ & 47.53 & 4348 & 7.61 & & & \\
\hline \multirow[t]{2}{*}{$\begin{array}{l}\text { HO } 30 \\
\text { Spatial }\end{array}$} & $\begin{array}{l}\text { Without } \\
\text { Siblings }\end{array}$ & 46.59 & 69 & 6.54 & \multirow[t]{2}{*}{0.79} & \multirow[t]{2}{*}{2.10} & \multirow[t]{2}{*}{$\begin{array}{l}\text { Not Significant } \\
\text { at } 0.05 \text { level }\end{array}$} \\
\hline & $\begin{array}{l}\text { With } \\
\text { Siblings }\end{array}$ & 48.26 & 4348 & 6.91 & & & \\
\hline \multirow[t]{2}{*}{$\begin{array}{l}\text { HO } 31 \text { Bodily } \\
\text { Kinesthetic }\end{array}$} & $\begin{array}{l}\text { Without } \\
\text { Siblings }\end{array}$ & 48.872 & 69 & 6.62 & 0.804 & 0.361 & \multirow[t]{2}{*}{$\begin{array}{l}\text { Not Significant } \\
\text { at } 0.05 \text { level }\end{array}$} \\
\hline & $\begin{array}{l}\text { With } \\
\text { Siblings }\end{array}$ & 48.43 & 4348 & 6.87 & & & \\
\hline \multirow[t]{2}{*}{$\begin{array}{l}\text { HO } 32 \\
\text { Musical }\end{array}$} & $\begin{array}{l}\text { Without } \\
\text { Siblings }\end{array}$ & 45.16 & 69 & 10.00 & \multirow[t]{2}{*}{1.212} & \multirow[t]{2}{*}{1.88} & \multirow[t]{2}{*}{$\begin{array}{l}\text { Not Significant } \\
\text { at } 0.05 \text { level }\end{array}$} \\
\hline & $\begin{array}{l}\text { With } \\
\text { Siblings }\end{array}$ & 47.44 & 4348 & 8.68 & & & \\
\hline
\end{tabular}


It was found that student 'with siblings' were better in Intra personal Intelligence and Spatial Intelligence whereas having or not having siblings did not have any effect on the rest of the intelligences.

\section{DISCUSSION}

Major findings of the present study are discussed in the context of previous researches to understand and reflect upon the drawn inferences more intensively and realistically.

\section{Working Status of Parents}

It was found that the children belonging to the family, in which both mother and father are working, were found better in Linguistic intelligence and bodily kinesthetic intelligence than children belonging to the family in which only one of the parents is working. Rest of the intelligences including logical mathematical, interpersonal, intrapersonal, bodily kinesthetic, spatial, musical and naturalist intelligence were not affected by working status of the parents. But, Patel (2014) and Govindan (2014), found that Intrapersonal intelligence was higher in the students of both working parents than single working parents.

\section{Parents' Qualification}

It was found that the students belonging to the family of Graduate parents were better than the students belonging to the Non Graduate Parents in Linguistic intelligence. This finding was supported by Patel (2014) and Govindan (2014). But, the present study also inferred that the students belonging to the family of Graduate parents were better than the students belonging to the Non Graduate Parents in Intrapersonal intelligence. Although, no study was found confirming or contradicting this finding of the present study. It was also found that the students belonging to the family of Graduate parents were better than the students belonging to the Non Graduate Parents in Musical intelligence. Rest of the intelligences was not affected by qualification of the parents. But, Govindan (2014), found that Bodily kinesthetic, Linguistic, Logical Mathematical were higher in the students belonging to the family of Graduate parents than Non Graduate parents.

\section{Joint family and nuclear family}

It was found that the nature of family did not have any effect on any of the intelligences of students. Although, researcher expected that nature of family affects the socialization of a child and many other attributes of the personality that did not found matching. But Govindan (2014), contradicted these findings and found that intrapersonal intelligence of student $s$ belonging to joint family was more compared to nuclear family students.

\section{Staying With and Without Siblings}

It was found that the students 'with sibling' were better in Intrapersonal intelligence than students 'without siblings'. This finding was contradicted by Govindan (2014) and found reverse 
result. It was also found that the students 'with sibling' were better in Spatial intelligence than students 'without siblings'. Govindan (2014) and Patel (2014) did not find any effect of siblings on spatial intelligence of students. The rest of the intelligences were not found affected by status of with and without siblings of the students.

As a researcher looking at the findings it can be concluded that the environment affects the level of various intelligences of learners. Hence, proper steps taken, can help to enhance the intelligences of learners. Teachers should identify the intelligence tendencies of the learners and should offer variety in teaching learning experiences to address the need of learners with different potential.

\section{REFERENCES}

Gardner, H. (1993). Multiple Intelligences - Theory into Practice, New York: Basic Books

Gardner, H. (1999). Intelligences reframed: Multiple Intelligences for the 21st century. New York: Basic Books

Gogebakan, D. (2003), How Students' Multiple Intelligences differ in terms of Grade level and Gender, Middle East: An unpublished M.Sc. Dissertation, Middle East: Middle East Technical University.

Govindan, G. (2014), A Study of Multiple Intelligences of Secondary School Students, An unpublished M.Ed. Dissertation, Gandhinagar: Vakil Shri D.H. Patel Dept. of Education, Kadi Sarva Vishwavidyalaya

Gurcay, D. (2003), The effect of Multiple Intelligences based instruction on students' Physics achievement, An unpublished M.Sc. Dissertation, Middle East: Middle East Technical University.

Jamal, Al-Assaf \& Others, (2012), To verify the level of Multiple Intelligences of Social Studies teachers in Jordan, An un published Ph.D. Thesis, Jordan: Alia University

Jha, S. (2014), Effectiveness of Intrapersonal Intelligence Based Teaching Strategies on Academic Achievement of Secondary School Students, An unpublished M.Ed. Dissertation, Gandhinagar: Vakil Shri D.H. Patel Dept. of Education, Kadi Sarva Vishwavidyala

Jose, A. (2011), An analysis of academic achievement and Bodily-Kinesthetic intelligence of school children, A Ph.D. Thesis, Kerala: School of Physical Education and Sports Sciences, Kottayam: Mahatma Gandhi University

Joshi, K. (2014), A Study of Effectiveness of Linguistic intelligence Based Teaching Strategies on Academic Achievement of Secondary School Student, An unpublished M.Ed. Dissertation, Gandhinagar: Vakil Shri D.H. Patel Dept. of Education, Kadi Sarva Vishwavidyalaya

Kaur and Chhikara(2008). Assessment of Multiple Intelligences among Young Adolescents (1214 Years). Department of Human Development and Family Studies, 4, Haryana, India J. Hum. Ecol., 23(1): 7-11 (2008) http://www.krepublishers.com/02-Journals/JHE/JHE-23- 


\section{Effect of Family Variables on Multiple Intelligences of Secondary School Students of Gujarat State}

0-000-000-2008-Web/JHE-23-1-000-000-2008-Abst-PDF/JHE-23-1-007-08-1739-KaurG/JHE-23-1-007-08-1739-Kaur-G-Tt.pdf

Kesavan, P SO (2013), A study on emotional intelligence, Multiple Intelligences, socio economic status and academic achievement of higher secondary students, An unpublished Ph.D. Thesis, Kerala: Periyar University

Kshatriya, V.(2014), A study of effectiveness of Naturalist intelligence based teaching strategies on academic achievement of secondary school students, An unpublished M.Ed. Dissertation, Gandhinagar: Vakil Shri D.H. Patel Dept. of Education, Kadi Sarva Vishwavidyalaya

Kumari, P. (2014), Effectiveness of Spatial Teaching Strategies on Academic of Secondary School Students, An unpublished M.Ed. Dissertation, Gandhinagar: Vakil Shri D.H. Patel Dept. of Education, Kadi Sarva Vishwavidyalaya

Loori, A. (2005), Multiple Intelligences : A Comparative Study Between the Preferences of Male and Females, Baherin: Arabian Gulf University

Lynn, C. (2009), Replacing brick and mortar schools: the importance of adding Multiple Intelligences to virtual learning, An un published Ph.D. Thesis, Capella: Capella University.

Majee, C.(2014), Effectiveness of Logical - Mathematical Intelligence Based Teaching Strategies on Academic Achievement of Secondary School Students, An unpublished M.Ed. Dissertation, Gandhinagar: Vakil Shri D.H. Patel Dept. of Education, Kadi Sarva Vishwavidyalaya

Partani, S. (2011), Developing Multiple Intelligences Training Module for Preschool Teachers and its effect on Multiple Intelligences of Preschool Children (3-4 years). An Unpublished Post Graduate Dissertation of Department of Human Development, Mumbai: S.N.D.T women's university

Patel, N.(2014), A Study of Multiple Intelligences of Secondary School Students, An unpublished M.Ed. Dissertation, Gandhinagar: Vakil Shri D.H. Patel Dept. of Education, Kadi Sarva Vishwavidyalaya

Ranade, M. D. (2006). Computer-based Instruction on "Multiple Intelligences Approach to Teaching” Research Studies in Teacher Education. APERA Conference 28-30 November, 2006, HOngKong http://edisdat.ied.edu.hk/pubarch/b15907314/full_paper/1289153177.pdf

Razmjoo, S. (2008), Study the relationship between Multiple Intelligences and language proficiency, An unpublished thesis, Iran: Shiraj University

Rozario \& BotelHO (2003), Multiple Intelligences theory in English Language Teaching (ELT): An analysis of current textbooks, materials and teachers' perceptions, Ohio: An unpublished M.A. Dissertation of Ohio University

Sharifi, H.P. (2008). The Introductory study of Gardner's Multiple Intelligences theory, in the field of lesson subjects and the students' compatibility, Free Islamic (Azad-e Eslami) University. Rud-e-hen Branch, Rud-e-hen, Iran Quarterly Journal of Educational Innovations, No. 24,Spring 2008.http://www.sid.ir/en/VEWSSID/J_pdf/97420082401.pdf 
Sharma, P. (2014), Effectiveness of Musical Intelligence Based Teaching Strategies on Academic Achievement of Secondary School Students, An unpublished M.Ed. Dissertation, Gandhinagar: Vakil Shri D.H. Patel Dept. of Education, Kadi Sarva Vishwavidyalaya

Yadav, A. (2014), A study of effectiveness of inter-personal intelligence on academic achievement of secondary school students, An unpublished M.Ed. Dissertation, Gandhinagar: Vakil Shri D.H. Patel Dept. of Education, Kadi Sarva Vishwavidyalaya 